\title{
MEASUREMENTS OF OSCILLATIONS AND FLEXURE OF ICEBERGS*
}

\author{
by \\ A. Foldvik, T. Gammelsrød and Y. Gjessing \\ (Geofysisk Institutt, Universitetet i Bergen, 5014 Bergen, Norway)
}

\section{ABSTRACT}

During the Norwegian Antarctic Research Expedition 1978-79, direct measurements of oscillations were carried out on 15 icebergs using a tiltmeter with an accuracy of $\pm 10 \mu \mathrm{rad}$. The amplitude of the oscillations varied from zero to about $10^{3} \mu \mathrm{rad}$. The zero amplitude indicates that the berg was grounded and this was confirmed by echo-sounding from the ship. The observed oscillation periods ranged from 16 to $50 \mathrm{~s}$. The observed oscillation periods and the calculated values based on the dimensions and mean density of the bergs were compared and the results are discussed. The flexure of the berg was measured with a theodolite and stakes. Relative movements exceeding the accuracy of the system (1 $\mathrm{mm}$ over $1 \mathrm{~km}$ distance) were not observed.

\section{INTRODUCTION}

An iceberg drifting or being transported across the ocean experiences both oscillations (rigid body response) and vibrations (elastic body response) as a result of waves. Few in situ measurements of stability and shear force on icebergs have been carried out so far. Basmaci (1978) calculated the stability and strength of tabular icebergs and concluded that these bergs are stable both statically and dynamically under extreme sea conditions if their width exceeds their depth. If the thickness is more than some $100 \mathrm{~m}$ the berg will also have sufficient strength to resist breaking up. The Norwegian Antarctic Research Expedition 1978-79 made measurements on 24 tabular icebergs in the Weddell Sea. Measurements of oscillations were carried out on 15 icebergs and observations of flexure were made on two. In this note we present the observations and compare them with theoretical results.

\section{METHODS AND OBSERVATIONS}

The instrument used for the oscillation measurements was a tiltmeter constructed by the Department of Geology, University of California, for the determination of variations of the surface inclination after periods of volcanic eruptions and earthquakes. The accuracy of this instrument is within $\pm 10 \mu \mathrm{rad}$, and it is $1.2 \mathrm{~m}$ long. It was placed on a horizontal wooden bar on the surface of the berg, normal to one of the assumed principal axes of rotation. The period of oscillation was obtained by reading the inclination every $2 \mathrm{~s}$ during a 5 to 10 minute period. The amplitude of the oscillations ranged from zero to $10^{3} \mu \mathrm{rad}$. A constant surface inclination indicated that the berg was grounded and this was confirmed by echo-sounding of the water depth. The oscillation period ranged from 16 to $50 \mathrm{~s}$ (Table I).

Flexure measurements were carried out on two bergs. Stakes were lined up across the berg and their relative vertical movements were measured by theodolite. The accuracy of the system was $1 \mathrm{~mm}$ over $1 \mathrm{~km}$ distance.

The height $h$ of the berg above sea-level was calculated from altimeter readings on the berg and on the ship, or by measurements with sextant and ship's radar. The total thickness $Z$ of the berg was determined by the observed height $h$ from the balance of weight and buoyancy.

$$
\underset{-(z-h)}{\mathcal{S}_{0}^{\rho} \rho_{\mathrm{w}}(z) \mathrm{d} z} \underset{-(Z-h)}{=\int^{h} \rho_{\mathrm{i}}(z) \mathrm{d} z} .
$$

Here $\rho_{w}$ and $\rho_{i}$ denote the densities of sea-water and ice, respectively. Introducing the average densities $\bar{\rho}_{\mathrm{w}}$ and $\bar{\rho}_{\mathrm{i}}$ we obtain

$$
\frac{h}{Z}=1-\frac{\bar{\rho}_{i}}{\bar{\rho}_{\mathbf{W}}}=1-\alpha .
$$

Direct measurements of the total thickness of the iceberg were carried out by radio echosounding on five bergs (Orheim 1980). The ratio $h / z$ varied from 0.14 to 0.17 with a mean value of 0.15 , and thus for a homogeneous iceberg

$$
Z=6.7 \mathrm{~h} \text {. }
$$

The horizontal dimensions of the berg (Table I) were calculated from the time and speed of the helicopter crossing the berg.

\section{RESULTS AND DISCUSSION}

F1exure measurements were carried out on two bergs with an estimated thickness of 400 and $120 \mathrm{~m}$. The amplitude of oscillation of the latter was $\pm 1.2 \mathrm{~m} \mathrm{rad}$ corresponding to vertical displacements of $\pm 0.40 \mathrm{~m}$ at the edge of the 
iceberg. Relative movements of the stakes exceeding the accuracy of the system $(1 \mathrm{~mm}$ over $1 \mathrm{~km}$ distance) were not observed. However, flexure beyond the capability of the measuring system may occur. Due to the elastic properties of the snow layer on the top of the berg, any sma11 flexure in the ice will decrease in the surface layer. Thus, to a good approximation, the berg can be regarded as a rigid body. The period of rigid-body oscillation of a rectangular and homogeneous tabular iceberg can be calculated from the formula

$$
T_{j}=2 \pi\left[\frac{\alpha Z\left(L_{j}^{2}+Z^{2}\right)}{g\left(L_{j}^{2}-6 \alpha(1-\alpha) Z^{2}\right)}\right]^{\frac{1}{2}},
$$

where $L_{i j}$ denotes the horizontal dimensions of the berg and $g$ the gravity. For $L_{j}<0.77 z$ the denominator in (4) is zero or negative, indicating unstable conditions. $L . j \gg>2$ gives a minimum period of oscillation,

$$
T_{\min }=2 \pi\left(\frac{\alpha Z}{g}\right)^{\frac{1}{2}}
$$

Figure 1 shows the observed values of $T$ as a function of the total thickness of the icebergs. The solid lines give the theoretical values for $L / Z$ according to Equation (4). The main features of Equation (4) are verified by the observations, viz. a general increase of the oscillation period with increasing thickness of the berg and also a tendency towards a reduction of the oscillation period with increasing length of the berg. However, the scatter is considerable and also some observation periods are shorter than the theoretical minimum given by Equation (5).

TABLE I. CALCULATED DIMENSIONS AND OBSERVED PERIODS OF OSCILLATION OF ICEBERGS

Dimensions of iceberg Period of oscillation (m) (s)

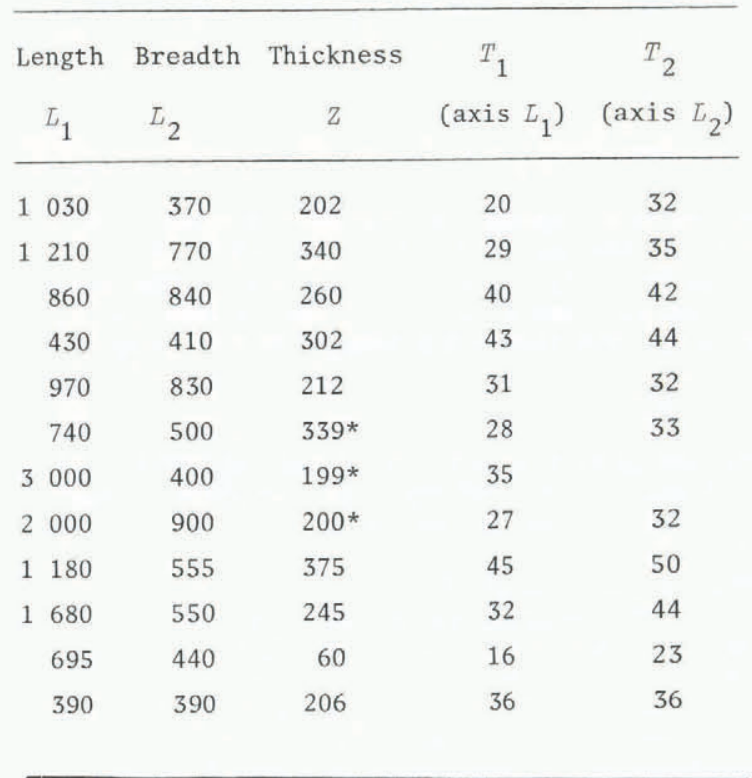

*Direct measurements



Fig.1. Observed and calculated periods of oscillation of icebergs as a function of total thickness.

Several factors may account for the discrepancy. Some of the discrepancy in Figure 1 may be due to the interpretation of the observations. A regular rectangular berg with horizontal dimensions $L_{1}$ and $L_{2}$ will oscillate around its principal horizontal axis with periods $T_{1}$ and $T_{2}$, respectively. In most cases these two periods were readily identified. However, bergs are not of regular rectangular shape, and it is likely that the record in some cases contains both $T_{1}$ and $T_{2}$. For example, the combination period $2 T_{1} T_{2} /\left(T_{1}+T_{2}\right)$ may have been accepted as a principal period due to the relatively short time of observation.

The theoretical curves are valid for a homogeneous iceberg with the observed value $\alpha=0.85$. This means that the density of the top layer is overestimated and also the moment of inertia of the berg. A correction for the density distribution would tend to reduce the theoretical oscillation periods in Figure $1 . \mathrm{A}$ correction of $T$ taking into account the density of the top layer will depend on the age of the berg, since normally the top-layer density increases in time due to melting and re-freezing. The problem of correlating the observations with a simple theory based on the geometry of the berg is further complicated by the unknown added mass of sea-water taking part in the oscillation. In addition to a damping effect this added mass will increase the inertia of the oscillating system and increase the period of oscillation.

\section{REFERENCES}

Basmaci Y 1978 Strength of icebergs during transport. In Husseiny A A (ed.) Iceberg utilization, Proceedings of the first International Conference, Ames, Iowa, 1977. New York, Pergamon Press: 191-198

Orheim 01980 Physical characteristics and life expectancy of tabular Antarctic icebergs. Annals of Glaciology (1): $11-18$ 